\title{
Directional Moving Target Indication: A Novel SAR Ambiguity Function for Traffic Monitoring
}

\author{
Paulo Marques ${ }^{1,2}$ \\ ${ }^{1}$ Instituto de Telecomunicações, Instituto Superior Técnico, Torre Norte, Piso 10, Av. Rovisco Pais, 1, 1049-001 Lisboa, Portugal \\ ${ }^{2}$ Department of Electronics and Telecommunications and Computer Engineering, Instituto Superior de Engenharia de Lisboa, \\ 1959-007 Lisboa, Portugal
}

Correspondence should be addressed to Paulo Marques, pmarques@isel.pt

Received 11 March 2011; Revised 1 November 2011; Accepted 19 December 2011

Academic Editor: Jiri Jan

Copyright (c) 2012 Paulo Marques. This is an open access article distributed under the Creative Commons Attribution License, which permits unrestricted use, distribution, and reproduction in any medium, provided the original work is properly cited.

\begin{abstract}
This paper presents and evaluates a novel SAR ambiguity function for traffic monitoring. The novelty consists in introducing the capability to discriminate targets moving in a predefined direction of interest, reducing the contribution of traffic moving in undesired directions. Experimental results show that the modified SAR ambiguity function provides better results than the traditional methodology and may, therefore, be useful for civil traffic monitoring using single-channel synthetic aperture radar data.
\end{abstract}

\section{Introduction}

Many recent publications address the problem of civil traffic monitoring using spaceborne and airborne Synthetic Aperture Radar (SAR) [1-8]. The main reasons for this growing attention are its all weather capability and large spatial coverage, which contrasts with those of conventional traffic monitoring sensors. The main conventional traffic sensors are stationary, using inductive or optical technologies. Although these kind of sensors are able to provide permanent coverage of the traffic situation, they can only perform local measurements. Moreover, in the case of the optical systems, they depend heavily on the sight conditions. A space-borne or airborne SAR system contrasts with the conventional ones, being able to cover large areas in short time obtaining data independently of the weather conditions. However, this kind of system presents, typically, low repetition rates and, in the case of spaceborne platforms, small flexibility. Therefore, although one cannot consider a SAR-based traffic monitoring system as a true alternative to the conventional systems, it may be regarded as a complementary source of information. There are already some solutions [5-8] that illustrate the capabilities of such systems.

Although many strategies for moving target indication (MTI) and velocity estimation have recently been published, such as $[1,3,4,8-13]$, none of them has the built in capability to discriminate between targets with different directions. This discrimination capability may be a very important goal in traffic monitoring since the roads of interest (with known angles) have, typically, many roads nearby with different directions. These nearby roads may carry traffic that, otherwise, will be detected and may corrupt the estimation of the traffic parameters for the roads of interest.

Some modern SAR-based traffic processors, such as those described in [6-8], already use a priori information about the road network to perform traffic extraction. This information is mainly used to derive the image areas where, due to the displacement effect, the moving vehicles images are expected to appear. The distance of the detected objects to the road axes is also used to estimate the velocity based on the azimuth displacement effect.

Some ambiguities can, however, still arise if the area of interest has a dense road network. In this situation, the SAR ambiguity function proposed in this paper may prove useful since it is able to filter out the vehicles that are not moving in the roads under study. This will reduce the computational requirements of the overall system since there will be a smaller number of targets to process and to perform consistency checks.

This paper, which further elaborates on [14], presents a novel SAR ambiguity function, which is direction selective and is herein designated as Directional Moving Target 
Indication (DMTI). The proposed DMTI is an elaborated version of the SAR ambiguity function proposed in [15, Chapter 6.7], which is not direction selective. The obtained results show that the resulting algorithm is highly selective, having the capability to detect and estimate the velocity of targets traveling in a certain predefined direction, reducing the contribution from undesired moving objects in the target region. Moreover, under the same conditions, the developed methodology is able to obtain more accurate results than the classical one.

This paper is organized as follows. Section 2 reviews the basic theory behind the classical SAR ambiguity function. Section 3 presents the modifications necessary in order to make it selective with respect to a predefined direction of interest. Section 4 illustrates the effectiveness of the resulting DMTI using simulated and real data. Section 5 draws the conclusions.

\section{Classical Approach}

This section starts by establishing the notation and terminology. The classical approach for moving target indication (MTI) is reviewed. It is shown that the MTI can be achieved by a phase compensation of the received echo, which depends simultaneously on both velocity components of a moving target.

The considered scenario is presented in Figure 1, where, for simplicity, a single moving target is present. The moving target has coordinates $\left(x_{m}, y_{m}\right)$ when the platform is at position $u=0$, and velocity $\left(v_{r}, v_{a}\right)=(\mu V, b V)$ defined in the slant plane $(x, y)$. The platform velocity is $V$ and $(\mu, b)=$ $\left(v_{r} / V, v_{a} / V\right)$ is the target relative velocity with respect to the radar. Terms $\mu$ and $b$ denote the target relative slant-range velocity and cross-range velocity, respectively.

The echoed signal in the fast-time frequency domain $\omega$ and slow-time spatial domain $u$ is, after pulse compression, given by $[2,4]$

$$
S_{m}(\omega, u)=|P(\omega)|^{2} a(\omega, u) f_{m} e^{-j 2 k r(u)}
$$

where

$$
r(u)=\sqrt{X_{m}^{2}+\left(Y_{m}^{2}-\alpha u\right)^{2}}
$$

is the distance between the target and the SAR platform, $P(\omega)$ is the emitted pulse in the frequency domain, $a$ denotes the two-way antenna pattern, and $\alpha=\sqrt{\mu^{2}+\nu^{2}}$, where $v=1+b$. The target reflectivity $f_{m}$ is considered constant. Coordinates $X_{m}$ and $Y_{m}$ are, as shown in [2, 4], the socalled motion transformed coordinates of the moving target [9], [15, Chapter 6.7], which correspond to a rotated and scaled version of coordinates $\left(x_{m}, y_{m}\right)$, that is,

$$
\left[\begin{array}{l}
x_{m} \\
y_{m}
\end{array}\right]=\frac{1}{\alpha}\left[\begin{array}{cc}
\nu & -\mu \\
\mu & v
\end{array}\right]\left[\begin{array}{c}
X_{m} \\
Y_{m}
\end{array}\right] \text {. }
$$

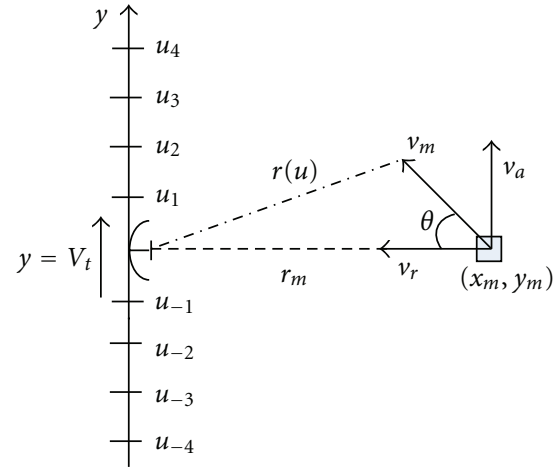

FIgURe 1: SAR Acquisition geometry.

Approximating distance $r(u)$ by a series expansion about $u=0$ and retaining only the terms through the quadratic results in

$$
r(u) \approx r_{m}-\frac{\mu x_{m}+v y_{m}}{X_{m}} u+\frac{\left(\mu^{2}+v^{2}\right)^{2}}{2 X_{m}} u^{2},
$$

valid for $|u| \ll X_{m}$.

In the slow-time frequency domain $k_{u}$, the linear term of (4) is responsible for a Doppler-shift, whereas the quadratic term is responsible for a Doppler-spread. If these effects are not considered in the focusing algorithm, the resulting SAR image shows the moving targets defocused and/or at wrong positions, depending on the motion direction. Basically, the Doppler-shift causes a misplacement of the moving targets in the azimuth direction, and the Doppler-spread causes their defocusing in the resulting image [16].

Soumekh presents in [15, Chapter 6.7] a moving target indicator, which is mainly based on the compensation of the quadratic phase component of (4). Since this component is responsible for a Doppler spread in the slow-time frequency domain, the search is done for the value of $\alpha$, which maximizes the energy concentration in the slow time frequency domain. Although the methodology is able to detect moving targets and to estimate their $\alpha$, which is related to the target velocity magnitude, it does not have the capability to discriminate between targets with different directions. This limitation is due to the coupling between the two velocity components in the quadratic phase term.

\section{Proposed Approach}

This section proposes a modification to the SAR ambiguity function described in the previous section in order to make it selective with respect to a certain direction of interest. It is shown that the amplitude modulation term due to the antenna pattern contains information, which can be related to the target direction. This information is used to filter out, in the conventional SAR ambiguity function, the targets moving in unwanted directions. This novel SAR ambiguity function is designated as Directional Moving Target Indication (DMTI).

In (4), it was shown that the phase information contained in the signal echoed by a moving target has a coupling 
between the two velocity components in the quadratic phase term. Therefore, methodologies that use only the phase information of the data acquired with a single-channel SAR are not able to discriminate the moving targets direction. This limitation is usually coined in the literature as the blind angle ambiguity [17].

Figure 2 illustrates the aforementioned limitation using two moving targets with different velocities and directions. Although the velocity vectors and directions are different, the distance of both targets with respect to the radar is the same for each emitted pulse. Therefore, the phase term of the echoed signal from each moving target does not contain sufficient information regarding the velocity vector direction.

Notice, however, that the amplitude of the received signals $\left|s_{1}(u)\right|$ and $\left|s_{2}(u)\right|$ is a dilated and translated version of the antenna radiation pattern amplitude. This fact is formally demonstrated in [17], where it is shown that the amplitude modulation term due to the antenna pattern is

$$
A\left(\omega, k_{u}\right) \propto g^{2}\left[\frac{1}{2 v}\left(k_{u}-2 k \mu\right)\right],
$$

where $A\left(\omega, k_{u}\right)$ is the $2 \mathrm{D}$ Fourier transform of the amplitude modulation term due to the antenna pattern and $g$ is related to the 2D Fourier transform of the electric field at the antenna aperture. The antenna pattern is shifted and scaled by a quantity, which is related to the range velocity and crossrange velocity, respectively. Both terms can be related with the target direction, as shown next, therefore removing the blind angle ambiguity.

3.1. Directional MTI. If we consider the scenario presented in Figure 1, where the moving target velocity magnitude is $v_{m}$ and its direction is $\theta$, the relative slant-range velocity is

$$
\mu\left(v_{m}, \theta\right)=\frac{v_{m}}{V} \cos \theta,
$$

and the quantity related to the cross-range velocity is

$$
\nu\left(v_{m}, \theta\right)=1+\frac{v_{m}}{V} \sin \theta .
$$

Parameter $\alpha$ is thus computed by

$$
\alpha\left(v_{m}, \theta\right)=\sqrt{1+\frac{v_{m}}{V}\left(\frac{v_{m}}{V}+2 \sin \theta\right)} .
$$

The Doppler spread in (4) can, therefore, be compensated as function of $\left(v_{m}, \theta\right)$ and the unwanted targets can be filtered out, besides the static ground echoes, by using the antenna pattern function presented in (5), as follows:

$$
\begin{aligned}
s_{c}\left(\omega, u, v^{\prime}, \theta^{\prime}\right)= & s_{m}(\omega, u) e^{j 2 k \psi\left(u, v^{\prime}, \theta^{\prime}\right)} \\
& * a^{*}\left(\omega, u, v^{\prime}, \theta^{\prime}\right),
\end{aligned}
$$

where

$$
\psi\left(u, v^{\prime}, \theta^{\prime}\right)=\sqrt{X_{c}^{2}+\left[Y_{c}-\alpha\left(v^{\prime}, \theta^{\prime}\right) u\right]^{2}} .
$$

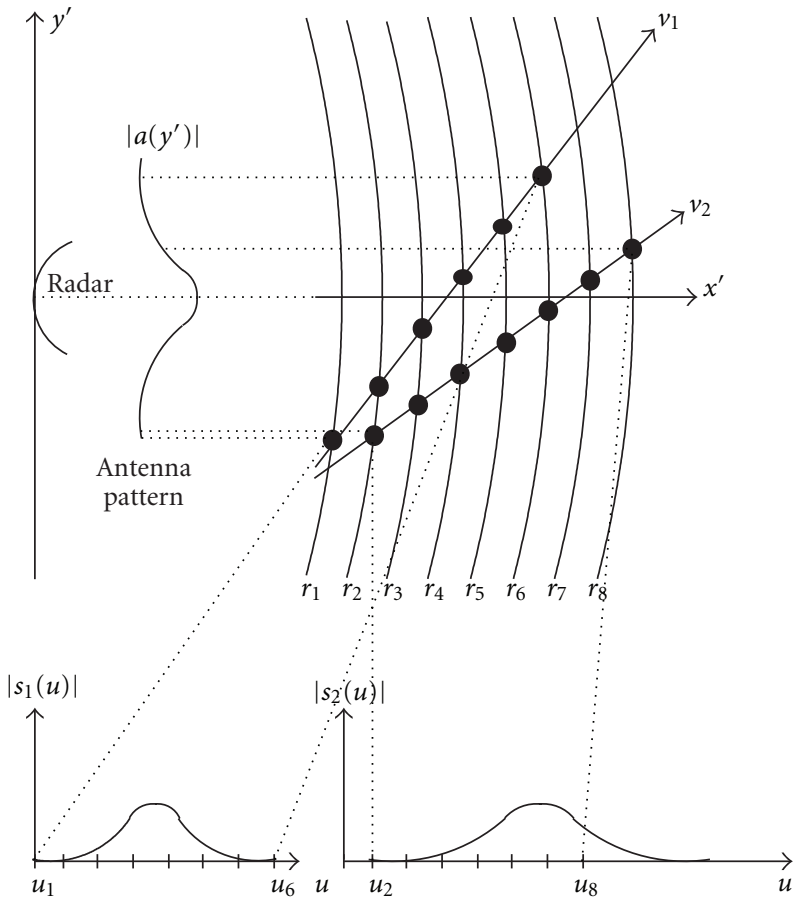

FIGURE 2: Two moving targets with different trajectories can induce the same phase on the received echoes $s_{1}(u)$ and $s_{2}(u)$. However, the amplitude information of these signals can be used to remove this ambiguity.

The central coordinates of the region under analysis are denoted by $X_{c}$ and $Y_{c}$. Parameters $\mu, \nu$, and $\alpha$ are computed by (6), (7), and (8).

For a moving target with parameters $\left(v_{m}, \theta_{m}\right) \approx\left(v^{\prime}, \theta^{\prime}\right)$, the Fourier transform of (9), with respect to slow-time $u$, will exhibit peaks, since the energy spread is compensated and the signal will not be filtered out by the term due to the antenna pattern. Therefore, the detection and estimation of moving targets with direction $\theta$ can be accomplished, in the slowtime frequency domain, by searching the absolute maxima of

$$
\begin{aligned}
\xi\left(\omega, k_{u}, v^{\prime}, \theta^{\prime}\right)= & A\left[\omega, k_{u}, \mu\left(v^{\prime}, \theta^{\prime}\right), v\left(v^{\prime}, \theta^{\prime}\right)\right] \\
& \times \operatorname{FT}_{(u)}\left[s_{m}(\omega, u) e^{j 2 k \sqrt{X_{m}^{2}+\left[Y_{m}-\alpha\left(v^{\prime}, \theta^{\prime}\right) u\right]^{2}}}\right],
\end{aligned}
$$

for a fixed $\omega$ and a range of velocities $v^{\prime}$ of interest.

In real scenarios due to the low SCR and to the possible presence of several strong static scatterers, the SAR ambiguity function for the moving targets of interest may be completely dominated by the clutter ambiguity signature. Moreover, the targets signature in the Fourier domain may partially, or fully, overlap with that from the clutter, depending on the direction of interest. Therefore, we use here a technique presented in [18] designated as digital spotlight. This process consists of focusing the target area with static ground parameters and cropping the region of interest. Although the moving targets will appear defocused, they span a region that is typically much smaller than the total illuminated scene. 
This way we reduce the contribution from most of the clutter and moving targets sufficiently apart from the region under study. The signature of the digitally spotlighted region is then resynthesized back to the desired domain as explained in [10] in order to apply the DMTI.

Range resolution is a measure of the resolvability of the targets in the range domain and will normally determine whether targets moving in close proximity will be detected as individual targets. The proposed scheme is able to detect the correct number of targets if their velocities $v_{m}$ are sufficiently apart in order to induce distinct peaks in (11). For this to occur, the velocity differences should be greater than the reciprocal of the digital spotlight region dimensions. Otherwise, if there are several targets with similar velocities and directions inside the same resolution cell, they will not be resolved and will be detected as a single target.

3.2. Computational Complexity. The computational complexity of the DMTI is now evaluated by using a strategy similar to that presented in [19, Chapter 12]. The approach consists in estimating the number of complex operations $\left(C_{\text {ops }}\right)$ for each major step of the algorithm. A complex operation is defined as one radix-2 FFT butterfly, which consists of ten floating point operations (four floating point multiplications and six floating point additions). An equal cost for multiplications and additions is assumed. Accordingly to [19, Chapter 12.2] and [20, Chapter 15], the following $C_{\mathrm{ops}}$ are accounted:

(i) FFT of size $N: C_{\mathrm{fft}} \approx N / 2 \log _{2} N\left[C_{\text {ops }}\right]$;

(ii) 2D FFT with dimension of $N_{x}$ by $N_{y}$ : $C_{\mathrm{fft} 2} \approx N_{x} N_{y} /$ $5 \log _{2} N_{x} N_{y}\left[C_{\text {ops }}\right]$;

(iii) complex multiplication: $C_{m} \approx 1\left[C_{\mathrm{ops}}\right]$;

(iv) complex-by-real multiplication: $C_{m r} \approx 0.5\left[C_{\mathrm{ops}}\right]$;

(v) 2D linear interpolation with size of $N_{x}$ by $N_{y}: C_{p} \approx$ $2 N_{x} N_{y}\left[C_{\text {ops }}\right]$.

The number of $C_{\text {ops }}$, just accounted, slightly overestimates the total number of operations for a given algorithm. This overestimation provides a margin for unaccounted machine cycles used in operations such as array index generation and memory access.

The DMTI algorithm requires the following main steps:

(a) one run of the wavefront reconstruction algorithm to image the target area with $N_{x}$ (slant-range) by $N_{y}$ (cross-range) samples;

(b) for each region where moving targets may be present:

(b1) digitally spotlight the region with size $N_{s x}$ (slant-range) by $N_{s y}$ (cross-range) and resynthesize its signature to the $(\omega, u)$ domain;

(b2) obtain reference signal $s_{m}(\omega, u)$, from the resynthesized signature, for a fixed $\omega$;

(b3) for a range of velocities $v$ and angles $\theta$ of interest, compute (6), (7), (8), and (11).

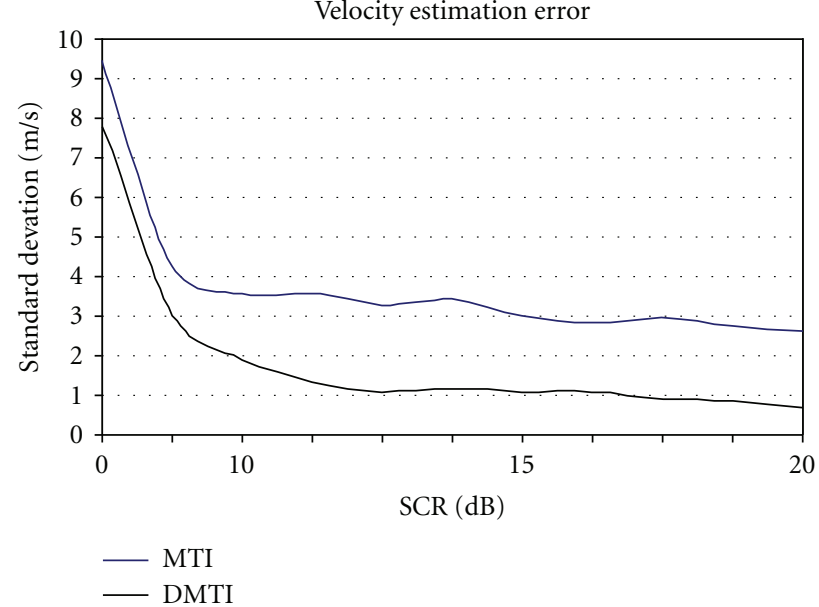

FIgURE 3: Monte Carlo results as a function of the SCR. The DMTI strategy outperforms the classical MTI by approximately $2 \mathrm{~dB}$.

Summing the number of $C_{\text {ops }}$ associated to each main step of the algorithm results in

$$
\begin{aligned}
C \approx & N\left(\frac{2}{5} \log _{2} N+1\right) \\
+ & N_{r}\left[N_{s}\left(\frac{2}{5} \log _{2} N_{s}+1\right)+N_{v} N_{\theta}\right. \\
& \left.\times\left(20+\sqrt{N_{s}}\left(3+\frac{1}{2} \log _{2} \sqrt{N_{s}}\right)\right)\right], \quad\left[C_{\text {ops }}\right]
\end{aligned}
$$

where $N \equiv N_{x} \times N_{y}$ and $N_{s} \equiv N_{s x} \times N_{s y}$; symbol $N_{r}$ denotes the number of regions where moving targets may be present, and $N_{\theta}$ and $N_{v}$ denote the number of discrete values for the search in $\theta$ and $v$ (step (b3)), respectively. To obtain a simpler expression, it was considered that $N_{x}=N_{y}$ and $N_{s x}=N_{s y}$.

As a numerical example, consider a target area of size $N=1024 \times 1024$ pixels, containing $N_{r}=20$ regions where moving targets may be present. Consider also there is a single direction $\theta$ of interest and that each digitally spotlighted region size is $N_{s}=100 \times 100$ pixels that $N_{v}=100$. In this situation, the algorithm requires, approximately, 12.5 millions of $C_{\text {ops }}$, which is accomplished in less than half a second in a current desktop computer with a processor speed of $2 \mathrm{GHz}$.

3.3. Monte Carlo Results. The proposed strategy is now compared with the classical approach. Figure 3 plots the standard deviation of the velocity obtained by Monte Carlo estimation for 20 experiments. The accuracy gain of the novel ambiguity function over the classical MTI is clear. Besides being able to filter out unwanted targets, and under the same SCR, the DMTI outperforms the classical approach by approximately $2 \mathrm{~dB}$. This improvement is mainly due to the filtering performed by the term related with the antenna pattern in (9). 
TABLE 1: Moving target parameters.

\begin{tabular}{lcc}
\hline Target & Velocity $(\mathrm{m} / \mathrm{s})$ & Direction $\left(^{\circ}\right)$ \\
\hline 1 & 5 & -86 \\
2 & 5 & -86 \\
3 & 10 & 94 \\
4 & 10 & 94 \\
5 & 12 & 90 \\
6 & 8 & 90 \\
\hline
\end{tabular}

TABLE 2: Mission parameters used in simulation.

\begin{tabular}{lc}
\hline Parameter & Value \\
\hline Carrier frequency & $9.6 \mathrm{GHz}$ \\
Chirp bandwidth & $250 \mathrm{MHz}$ \\
Altitude & $12 \mathrm{~km}$ \\
Velocity & $637 \mathrm{~km} / \mathrm{h}$ \\
Look angle & $15^{\circ}$ \\
Antenna radiation pattern & Raised Cosine \\
\hline
\end{tabular}

\section{Estimation Results}

The scheme proposed in the previous section is now tested with synthetic and real data. The synthetic data uses six moving targets with motion parameters such that the directional selectivity of the DMTI can be illustrated. The experiments with real data use clutter and targets from the Moving and Stationary Target Acquisition and Recognition (MSTAR) public collection [21]. The clutter is a scene from Hunstville-Alabama, and the targets are five ZIL-131 cargo trucks with simulated movement. With the goal of illustrating the accuracy gain of the DMTI over the classical MTI, Monte Carlo results are also presented.

4.1. Synthetic Data. The simulated scene contains six moving targets with motion parameters presented in Table 1 . The signal-to-clutter ratio (SCR) for each moving target is $20 \mathrm{~dB}$. Notice that the definition of SCR herein used corresponds to the ratio between the peak (squared magnitude) of the correctly focused moving target and the covariance of the background. Moving targets 1 to 4 are all moving on the same road. However, targets 1 and 2 move in the opposite direction of targets 3 and 4 . To illustrate the DMTI selectivity with respect to a direction of interest, targets 5 and 6 move in a road next to the first, but with a direction difference of $4^{\circ}$. This scenario is illustrated in Figure 4.

The misson parameters used in the simulation are presented in Table 2.

Figure 5 illustrates the obtained results using the proposed DMTI using $\theta=-86^{\circ}$ as the direction of interest. As can be seen, only two maximums are clearly visible, which correspond to targets 1 and 2 . The estimated velocities are $4.4 \mathrm{~m} / \mathrm{s}$ for target 1 and $4.8 \mathrm{~m} / \mathrm{s}$ for target 2 . The remaining moving targets do not produce energy concentration peaks, as expected.

Figure 6 shows the obtained results using $\theta=94^{\circ}$ as the direction of interest. The two maximums correspond

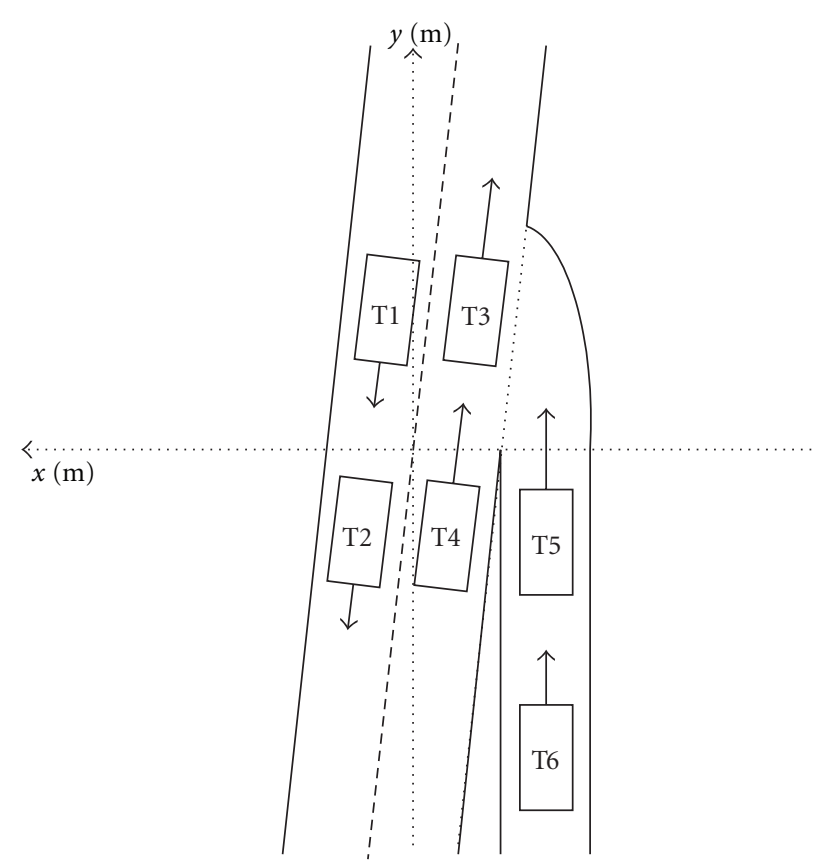

Figure 4: Simulation scenario.

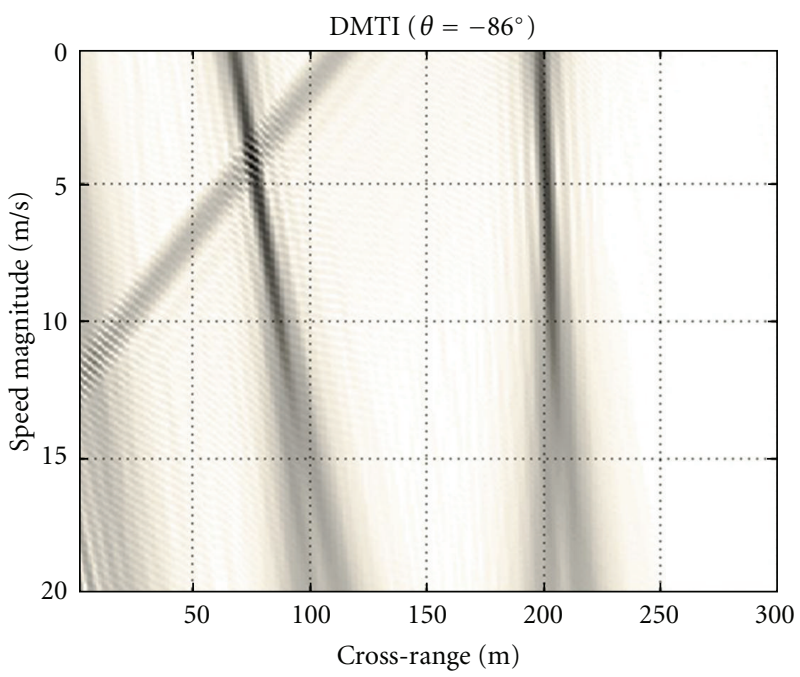

Figure 5: DMTI results for $\theta=-86^{\circ}$.

to targets 3 and 4 . The estimated velocities are $10.1 \mathrm{~m} / \mathrm{s}$ and $9.6 \mathrm{~m} / \mathrm{s}$, for target 3 and target 4 , respectively. This figure also presents two other potential contributions, which correspond to targets 5 and 6 since they have a very small direction difference of $4^{\circ}$. However, their contribution is attenuated and spreaded due to the filtering performed by the antenna pattern in (9). These results illustrate the direction selectivity of the proposed DMTI.

4.2. MSTAR Data. The proposed scheme is now applied to data supplied by the US Air Force Research Laboratory, from the Moving and Stationary Target Acquisition and Recognition (MSTAR) program [21]. The experiment uses clutter 


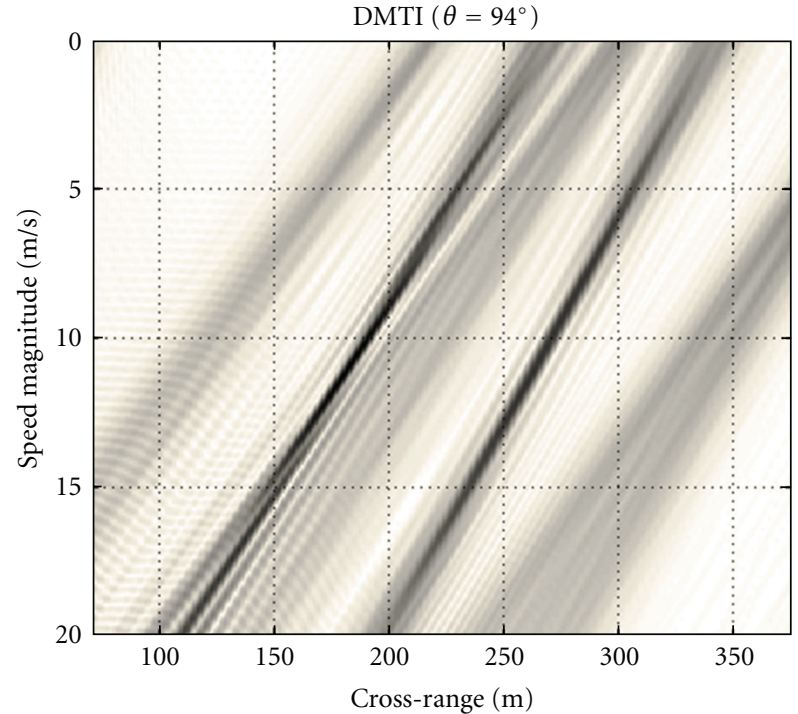

FIgURE 6: DMTI results for $\theta=94^{\circ}$.

from Huntsville-Alabama and five ZIL-131 with simulated movement. Figure 7 shows the optical and X-band images of the considered targets. To obtain a smaller dataset, a low-pass filtering to the original data and a decimation by a factor of 4 was applied in both directions. The mission parameters are listed in Table 2.

The moving target parameters are presented in Table 3. Target 5 is stationary, being used as reference target. The SCR is roughly set to $20 \mathrm{~dB}$.

The resulting data was focused using the wavefront reconstruction algorithm with static ground parameters. The obtained image is presented in Figure 8, where moving targets 3 and 4 appear defocused and misplaced. Targets 1 and 2 are mainly misplaced, as expected.

The area used to apply the DMTI contains, intentionally, the signature of all 5 targets. Using the DMTI with $\theta=-86^{\circ}$, we were able to detect and estimate the velocity only for those targets moving in the desired direction. As expected, only targets 3 and 4 are detected. The DMTI is able to filter out the remaining targets. The velocity estimation presents an error of $1.54 \mathrm{~m} / \mathrm{s}$ and $2.37 \mathrm{~m} / \mathrm{s}$, respectively, which are acceptable results. One should note, however, that in real situations the accuracy is expected to be lower since the estimation algorithm assumes that the moving target does not contain acceleration, pitch, or roll.

\section{Conclusion}

This paper presented a novel moving target indicator, which is selective with respect to a direction of interest. This selectivity may be useful for traffic monitoring in civil environments where the target area contains, simultaneously, traffic moving in several different directions, besides that of interest. It is shown that the amplitude modulation term due to the antenna pattern contains information, which can be related to the target direction. This information is used to filter out the undesired targets. The resulting SAR ambiguity

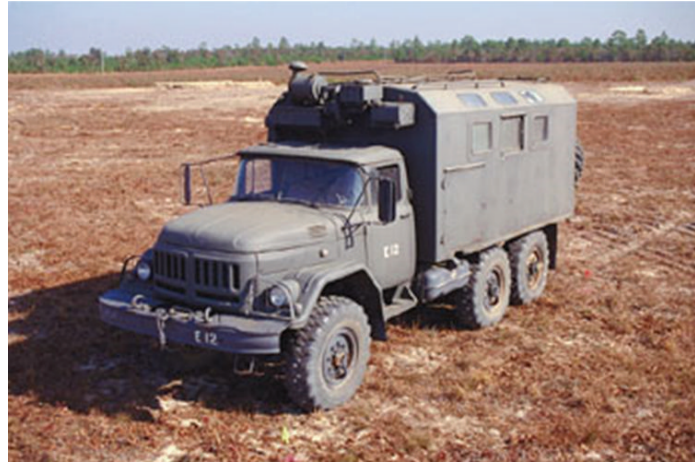

(a)

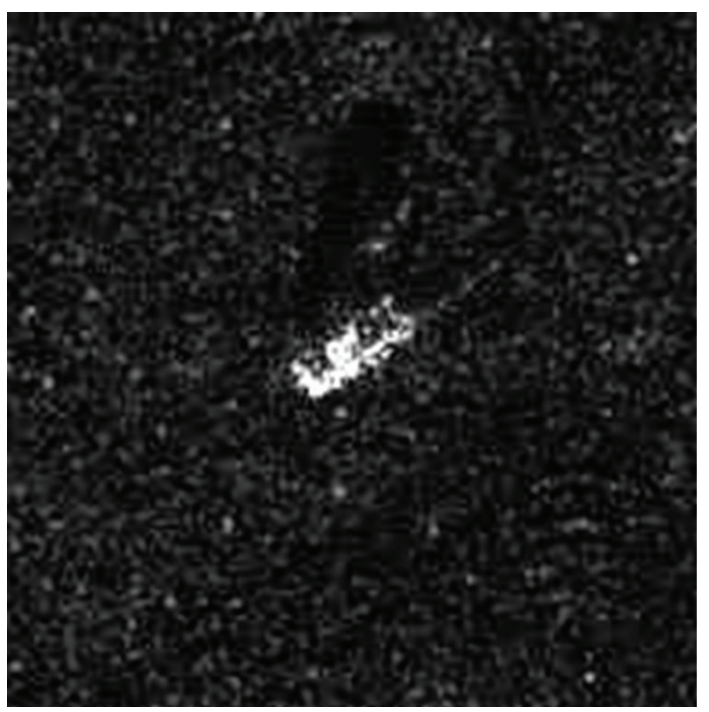

(b)

FIgURE 7: Visible (a) and X-band (b) images of ZIL-131 cargo truck. Data from MSTAR data public collection.

TABLE 3: ZIL 131 motion parameters.

\begin{tabular}{lcccc}
\hline Target & $x_{0}(\mathrm{~m})$ & $y_{0}(\mathrm{~m})$ & Velocity $(\mathrm{m} / \mathrm{s})$ & Direction $\left(^{\circ}\right)$ \\
\hline 1 & 90 & 279 & 5 & 10 \\
2 & 110 & 282 & 5 & 10 \\
3 & 75 & 245 & 40 & -86 \\
4 & 82 & 215 & 40 & -86 \\
5 & 55 & 275 & 0 & N.A. \\
\hline
\end{tabular}

function is herein designated as directional moving target indication (DMTI). An expression for the computational requirements of the global scheme was derived. It was shown that the requirements for realistic scenarios can be efficiently tackled. The methodology was tested using a combination of simulated and real data. The experiments used simulated and real data from the MSTAR public collection. With the goal of illustrating the accuracy gain of the DMTI over the classical MTI, Monte Carlo simulations were also presented. The obtained results show that the developed MTI, besides being selective, obtains more accurate results than those obtained by the classical method. The accuracy gain is mainly due to 


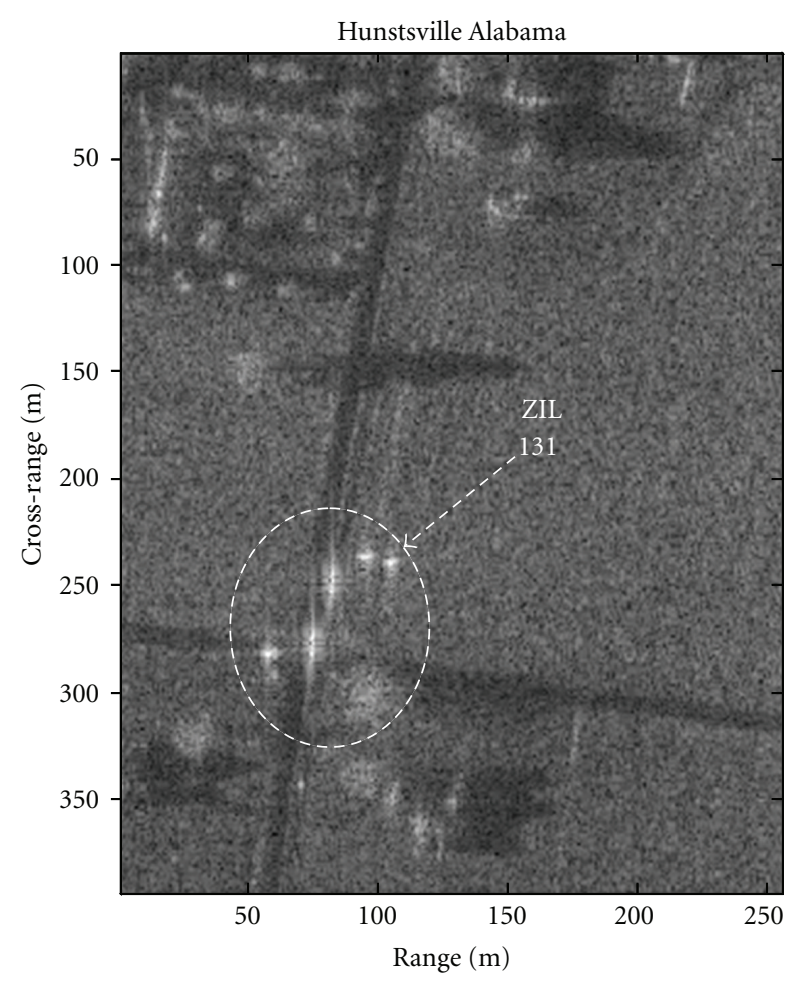

FIgURE 8: Target area focused with $\alpha=1$.

the filtering performed by the term related with the antenna pattern.

Provided that the SCR is better than $10 \mathrm{~dB}$, the proposed scheme is able to accurately detect and estimate the velocity of the targets traveling in a predefined direction, filtering out the contribution from the remaining targets. Furthermore, it has low computational requirements and can be used with single channel SAR data.

\section{Acknowledgments}

This paper was partially supported by the Portuguese Foundation for Science and Technology under Project PTDC/ EEA-TEL/71996/2006 and by European Project Development of Pre-operational Services for Highly Innovative Maritime Surveillance Capabilities (DOLPHIN) in the context of the Collaborative Project FP7-SPACE-2010-1, Reference: 263079. The author also wishes to acknowledge the Air Force Research Laboratory (AFRL) and the Defense Advanced Research Projects Agency (DARPA) for providing the MSTAR data.

\section{References}

[1] M. Delphine, C. Girtull, and J. Ender, "First experimental demonstration of GMTI improvement through antenna switching," in Proceedings of the 7th European Conference on Synthetic Aperture Radar (EUSAR '08), Friedrichshafen, Germany, June 2008.

[2] S. Suchandt, M. Eineder, H. Breit, and H. Runge, "Analysis of ground moving objects using SRTM/X-SAR data," ISPRS
Journal of Photogrammetry and Remote Sensing, vol. 61, no. 34, pp. 209-224, 2006.

[3] S. Hinz, D. Weihing, S. Suchandt, and R. Bamler, "Detection and velocity estimation of moving vehicles in high-resolution spaceborne synthetic aperture radar data," in Proceedings of the IEEE Computer Society Conference on Computer Vision and Pattern Recognition Workshops (CVPR '08), pp. 1-6, June 2008.

[4] D. Cerutti-Maori, J. Klare, A. R. Brenner, and J. H. G. Ender, "Wide-area traffic monitoring with the SAR/GMTI system PAMIR," IEEE Transactions on Geoscience and Remote Sensing, vol. 46, no. 10, pp. 3019-3030, 2008.

[5] S. Suchandt, H. Runge, H. Breit, U. Steinbrecher, A. Kotenkov, and U. Balss, "Automatic extraction of traffic flows using TerraSAR-X along-track interferometry," IEEE Transactions on Geoscience and Remote Sensing, vol. 48, no. 2, pp. 807-819, 2010.

[6] A. Laika, F. Meyer, S. Hinz, and R. Bamler, "Incorporating a priori knowledge into a moving vehicle detector for terraSARX data," in Proceedings of the 7th European Conference on Synthetic Aperture Radar (EUSAR '06), Dresden, Germany, June 2006.

[7] S. Hinz, F. Meyer, M. Eineder, and R. Bamler, "Traffic monitoring with spaceborne SAR-Theory, simulations, and experiments," Computer Vision and Image Understanding, vol. 106, no. 2-3, pp. 231-244, 2007.

[8] S. Chiu and M. V. Dragošević, "Moving target indication via RADARSAT-2 multichannel synthetic aperture radar processing," EURASIP Journal on Advances in Signal Processing, vol. 2010, Article ID 740130, 19 pages, 2010.

[9] P. A. C. Marques and J. M. B. Dias, "Velocity estimation of fast moving targets using a single SAR sensor," IEEE Transactions on Aerospace and Electronic Systems, vol. 41, no. 1, pp. 75-89, 2005.

[10] P. A. C. Marques and J. M. Bioucas Dias, "Moving targets processing in SAR spatial domain," IEEE Transactions on Aerospace and Electronic Systems, vol. 43, no. 3, pp. 864-874, 2007.

[11] J. R. Fienup, "Detecting moving targets in SAR imagery by focusing," IEEE Transactions on Aerospace and Electronic Systems, vol. 37, no. 3, pp. 794-809, 2001.

[12] M. Kirscht, "Detection, velocity estimation and imaging of moving targets with single-channel SAR," in Proceedings of the 7th European Conference on Synthetic Aperture Radar (EUSAR '98), pp. 587-590, 1998.

[13] M. Kirscht, "Detection and imaging of arbitrarily moving targets with single-channel SAR," in Proceedings of the IEEE Radar Conference (RADAR '02), pp. 280-285, October 2002.

[14] P. Marques, "A novel SAR ambiguity function for traffic monitoring," in Proceedings of the 8th European Conference on Synthetic Aperture Radar (EUSAR '10), pp. 309-312, Aachen, Germany, June 2010.

[15] M. Soumekh, Synthetic Aperture Radar Signal Processing with MATLAB Algorithms, Wiley-Interscience, 1999.

[16] K. Ouchi, "On the multilook images of moving targets by synthetic aperture radars," IEEE Transactions on Antennas and Propagation, vol. 33, no. 8, pp. 823-827, 1985.

[17] J. M. B. Dias and P. A. C. Marques, "Multiple moving target detection and trajectory estimation using a single SAR sensor," IEEE Transactions on Aerospace and Electronic Systems, vol. 39, no. 2, pp. 604-624, 2003.

[18] M. Soumekh, "Reconnaissance with ultra wideband UHF synthetic aperture radar," IEEE Signal Processing Magazine, vol. 12, no. 4, pp. 21-40, 1995. 
[19] W. Carrara, R. Goodman, and R. Majewski, Spotlight Synthetic Aperture Radar: Signal Processing Algorithms, Artech House, 1995.

[20] S. Kay, Modern Spectral Estimation, Prentice-Hall, Englewood Cliffs, NJ, USA, 1988.

[21] T. Ross, S. Worrell, V. Velten, J. Mossing, and M. Bryant, "Standard SAR ATR evaluation experiments using the MSTAR public release data set," in Algorithms for Synthetic Aperture Radar Imagery $V$, vol. 3370 of Proceedings of SPIE, Orlando, Fla, USA, 1998. 

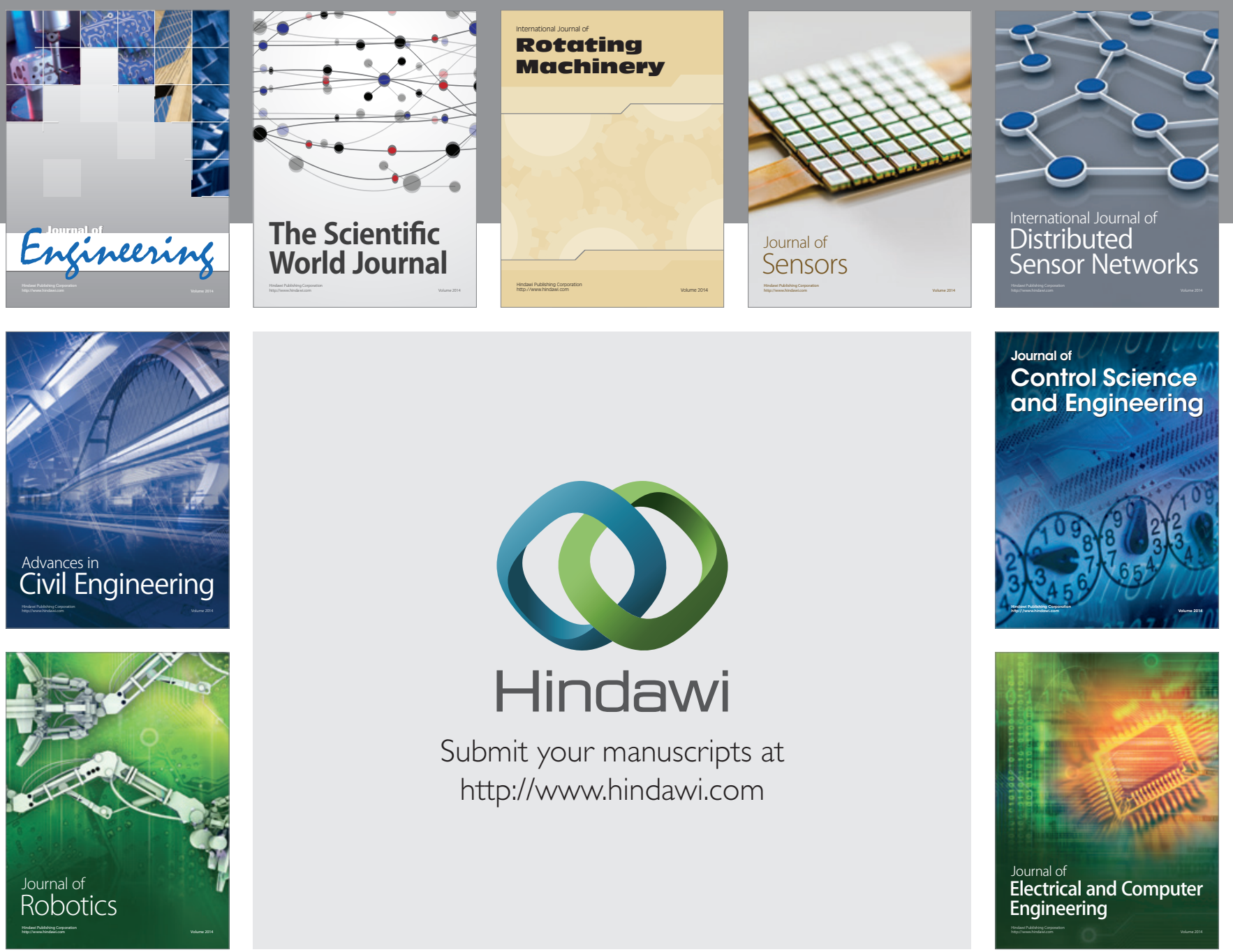

Submit your manuscripts at

http://www.hindawi.com
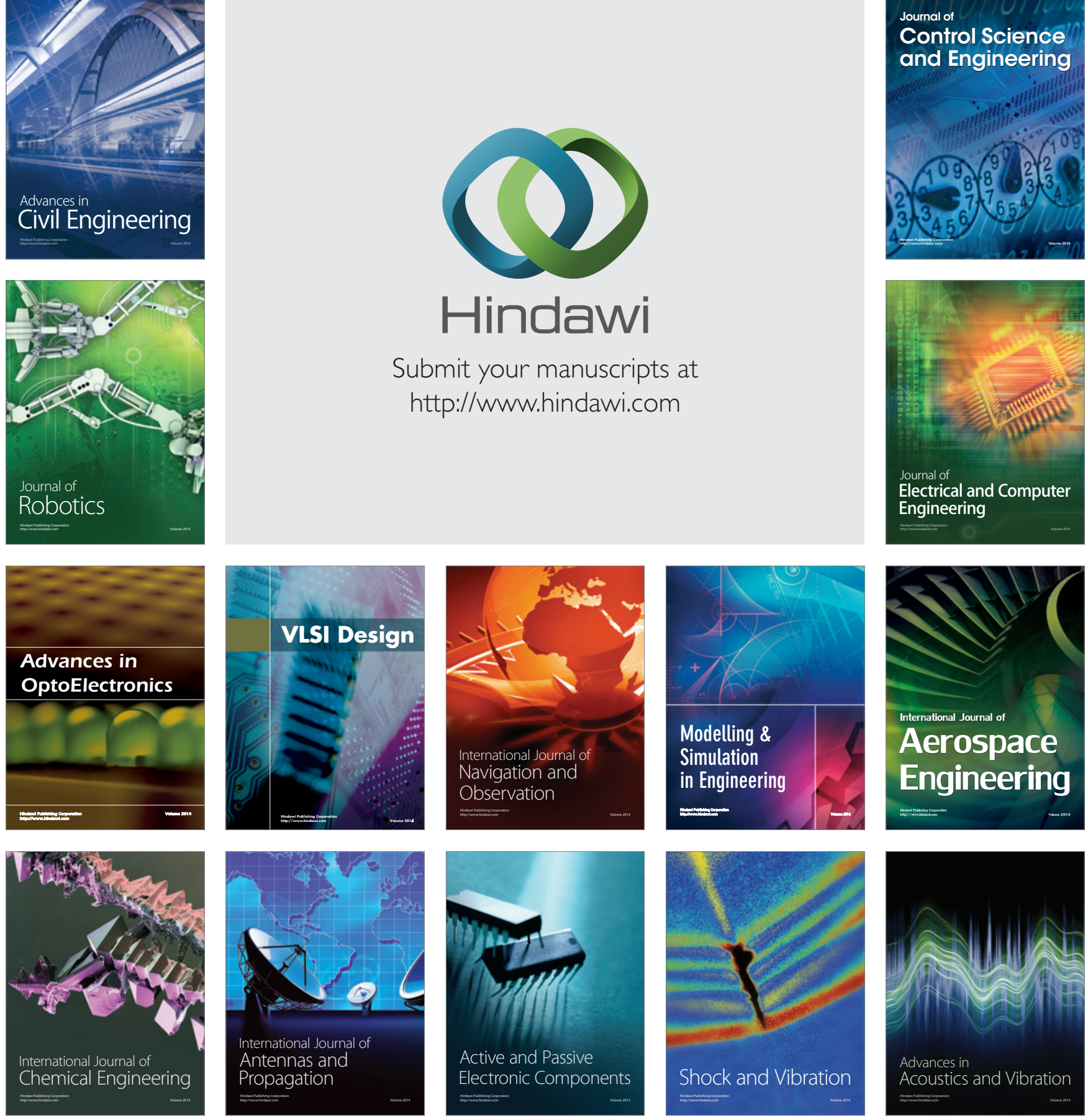